\title{
A Method to Evaluate Fetal Erythropoiesis from Postnatal Survival of Fetal RBCs
}

\author{
Denison J. Kuruvilla, ${ }^{1}$ John A. Widness, ${ }^{2}$ Demet Nalbant, ${ }^{2}$ Robert L. Schmidt, ${ }^{2}$ \\ Donald M. Mock, ${ }^{3,4}$ and Peter Veng-Pedersen ${ }^{1,5}$
}

Received 23 March 2015; accepted 4 May 2015; published online 28 May 2015

\begin{abstract}
Fetal RBCs are produced during a period of very rapid growth and stimulated erythropoiesis under hypoxic intrauterine conditions. Fetal RBC life span varies with gestational age (GA) and is shorter than that in healthy adults. Due to the special kinetic properties of life span-based survival of human RBCs, a mathematical model-based kinetic analysis of the survival of fetal RBCs shortly after birth provides a unique opportunity to "look backward in time" to evaluate fetal erythropoiesis. This work introduces a novel method that utilizes postnatal in vivo RBC survival data collected within 2 days after birth to study both nonsteady-state (non-SS) in utero RBC production and changing fetal RBC life span over time. The effect of changes in erythropoiesis rate and RBC life span and the effect of multiple postnatal phlebotomies on the RBC survival curves were investigated using model-based simulations. This mathematical model, which considers both changes in the rate of erythropoiesis and RBC life span and which accurately accounts for the confounding effect of multiple phlebotomies, was applied to survival curves for biotin-labeled RBCs from ten anemic very low birth weight preterm infants. The estimated mean fetal RBC production rate scaled by body weight was $1.07 \times 10^{7} \mathrm{RBCs} / \mathrm{day} \mathrm{g}$, and the mean RBC life span at birth was 52.1 days; these values are consistent with reported values. The in utero $\mathrm{RBC}$ life span increased at a rate of 0.51 days per day of gestation. We conclude that the proposed mathematical model and its implementation provide a flexible framework to study in utero non-SS fetal erythropoiesis in newborn infants.
\end{abstract}

KEY WORDS: cord blood RBCs; fetal erythropoiesis; fetal RBC life span; fetal RBC production; red blood cells.

\section{INTRODUCTION}

Erythropoietic status in newborn infants is determined by two phases: the fetal erythropoiesis before birth and neonatal erythropoiesis after birth. The former is important in understanding the mechanisms involved in red blood cell (RBC) production in the hypoxic intrauterine environment, while the latter provides a better understanding of the newborn infant's ability to compensate for the expected decline in postnatal erythropoietic activity that predictably results from the increased oxygen availability and resulting downregulation of erythropoietin synthesis and release (1-3). In anemic preterm infants, the latter also provides information about the

\footnotetext{
$\overline{{ }^{1} \text { Department of Pharmaceutical Sciences and Experimental Thera- }}$ peutics, College of Pharmacy, University of Iowa, 115 S. Grand Ave. S227, Iowa City, Iowa 52242, USA.

${ }^{2}$ Department of Pediatrics, College of Medicine, University of Iowa, Iowa City, Iowa, USA.

${ }^{3}$ Departments of Biochemistry and Molecular Biology, University of Arkansas for Medical Sciences, Little Rock, Arkansas, USA.

${ }^{4}$ Department of Pediatrics, University of Arkansas for Medical Sciences, Little Rock, Arkansas, USA.

${ }^{5}$ To whom correspondence should be addressed. (e-mail: veng@uiowa.edu)
}

critically ill infant's ability to compensate for blood loss due to the multiple clinical phlebotomies that commonly results from required neonatal care. Determining the postnatal erythropoiesis rate would also help in assessing and improving the efficacy of strategies such as erythropoietin therapy that are aimed at reducing or eliminating $\mathrm{RBC}$ transfusions.

The RBCs present at birth have been formed during the latter part of fetal life (4). During this period, the fetus experiences rapid growth, and as a result, the rate of RBC production is high (5). The progressive increase in hemoglobin $\mathrm{Hb}$ concentration and erythrocyte content in whole blood that is observed during the course of intrauterine development provides ample evidence that the RBC production increases with time in utero, leading to high $\mathrm{Hb}$ values at birth (6-8).

Past studies indicate that the RBC life span of a term newborn infant is only about two thirds (60 to 80 days) that of a healthy adult (9). Further, RBC life span is even shorter in preterm infants than in full-term infants and appears to decrease with birth weight (2). Likewise, in ovine fetuses, the fetal RBC life span increases with gestational age (GA) and is less than that in the adult (10). Since the Hb level at any time is dependent on both the production and the survival of RBCs, a better understanding of the intrauterine changes in fetal RBC life span is crucial in evaluating fetal erythropoiesis in newborn infants. 
Several studies have utilized human fetal RBCs isolated from either umbilical cord or placentas at delivery to study in utero erythropoiesis $(2,4,11,12)$. These studies primarily focused on estimating the fetal $\mathrm{RBC}$ life span and typically comparing fetal RBC life span to adult RBC life span. However, these studies did not evaluate the nonsteady-state (non-SS) conditions under which the cord blood RBCs were produced. This simplification may have confounded previous conclusions. Because cord blood RBCs were formed during the latter part of fetal life and their survival is life span based, the mathematical analysis of the survival curves of these RBCs provides a unique opportunity to "look backward in time" for evaluating the phase of fetal erythropoiesis in newborn infants.

The objectives of the present study are the following: (1) to present a novel method that utilizes cord blood or infant blood RBCs collected within the first days after birth to study both the non-SS in utero RBC production and the changes in in utero RBC life span over time and (2) to apply this method to in vivo RBC disappearance curves of umbilical cord RBCs from critically ill very low birth weight (VLBW) preterm infants tracked via a biotin label.

\section{MATERIALS AND METHODS}

\section{The Mathematical Model}

Intrauterine Growth. Intrauterine growth was estimated using birth weight as a function of GA data of Arbuckle et al. (13). These data from over one million live births represent one of the largest live birth datasets available that includes GA (13). The 50th birth weight percentile-GA data was digitally extracted for male singleton, female singleton, male twin, and female twin live births. A fourth-order polynomial function, which provided the best fit to each dataset, was used to model the change in birth weight with GA (Eq. 1 and Fig. 1). It was assumed that the data generated from the birth weights of preterm infants was representative of intrauterine growth of fetuses remaining in utero up to the time of birth. For GA less than that included in this dataset (i.e., less than 154 days), an exponential function (Eq. 1 and Fig. 1) was used for estimating intrauterine growth. The in utero body weight, BW(GA), can be expressed as (Fig. 1):

$\mathrm{BW}(\mathrm{GA})=\left\{\begin{array}{cc}A \cdot \mathrm{GA}^{4}+B \cdot \mathrm{GA}^{3}+C \cdot \mathrm{GA}^{2}+D \cdot \mathrm{GA}+E & \mathrm{GA}>154 \\ M \cdot\left(e^{\gamma \cdot \mathrm{GA}_{-}}-1\right) & 0<\mathrm{GA} \leq 154\end{array}\right.$

where GA is the gestational age of the infant measured in days and $A, B, C, D, E, M$, and $\gamma$ are the parameters listed in Table I.

In Utero Erythropoiesis Rate. The in utero erythropoiesis rate, $R(t)$, is considered to be proportional to the body weight and accordingly is expressed as:

$$
R(t)=k \cdot \mathrm{BW}(t+\mathrm{GA}) \quad t \leq 0
$$

where $B W(t)$ is the in utero body weight of the infant at time $t, k$ is a scaling/proportionality factor, and $t$ is the time relative to the time of birth $(t=0)$. At the time of birth, i.e., when $t=0$, $R(0)$ will be proportional to $B W(G A)$, and for any time $t$ thereafter, $R(t)$ will be proportional to the infant body weight at time $t+G A$. For the specific case that assumes a life span-based

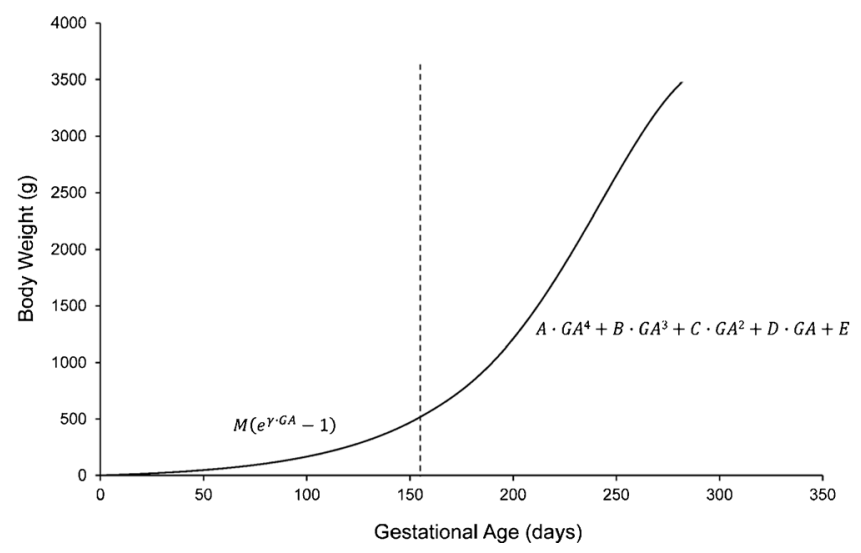

Fig. 1. Intrauterine growth of newborn infants. The birth weight $v s$. GA data (50th birth weight percentile) extracted from Arbuckle et al. (13) was used to approximate the intrauterine growth for male singleton, female singleton, male twin, and female twin infants. For GA greater than 154 days (dashed line), each dataset was separately fitted with a fourth-order polynomial function (Eq. 1). For GA less than that included in this birth cohort, i.e., less than 154 days, an exponential function (Eq. 1) was used for extrapolation of intrauterine growth. The values of parameters $A, B, C, D, E, M$, and $\gamma$ are listed in Table I

disposition with a fixed in utero $\mathrm{RBC}$ life span (i.e., no change in in utero $\mathrm{RBC}$ life span with time), the number of RBCs produced in utero present at the time of birth is given by:

$n(0)=\int_{-L(0)}^{0} R(t) \mathrm{d} t$

In Eq. 3, the in utero RBC production rate is integrated from $-L(0)$ to 0 , where $L(0)$ represents the fixed fetal RBC life span that is also equal to the RBC life span at the time of birth $(t=0)$. To consider the more complex case of variable in utero $\mathrm{RBC}$ life span, the lower integration limit in Eq. 3 has to be modified. Previously published data in ovine fetuses indicate that the fetal RBC life span increases approximately linearly with GA (10). Considering a similar case in humans, the linear change in in utero fetal RBC life span with time can be expressed as:

$L(t)=L(0)+\alpha t \quad t \leq 0$

where $\alpha$ is the slope parameter that describes the rate of change in fetal RBC life span with time. Let $x$ be defined as an intermediate variable defining the integration limits for the number of RBCs produced in utero, which are eliminated after birth, obtained as follows:

$x=t-L(x) \quad x \leq 0$

From Eqs. 4 and 5, we get:

$x=t-L(0)-\alpha x$

$x=\frac{t-L(0)}{1+\alpha}$ 
Table I. Parameter Estimates Obtained by Fitting Eq. 2 to Birth Weight-GA Data Extracted from Arbuckle et al. (13)

\begin{tabular}{lllll}
\hline & Male, singleton & Female, singleton & Male, twin \\
\hline$A\left(\mathrm{~g} \cdot \mathrm{day}^{-4}\right)$ & $-1.21 \times 10^{-5}$ & $-1.60 \times 10^{-5}$ & $-9.09 \times 10^{-6}$ & Female, twin \\
$B\left(\mathrm{~g} \cdot \mathrm{day}^{-3}\right)$ & $9.37 \times 10^{-3}$ & $1.28 \times 10^{-2}$ & $6.72 \times 10^{-3}$ & $-1.28 \times 10^{-5}$ \\
$C\left(\mathrm{~g} \cdot \mathrm{day}^{-2}\right)$ & -2.53 & -3.66 & -1.73 & -2.87 \\
$D\left(\mathrm{~g} \cdot \mathrm{day}^{-1}\right)$ & $3.0 \times 10^{2}$ & $4.61 \times 10^{2}$ & $1.98 \times 10^{2}$ & \\
$E(\mathrm{~g})$ & $-1.30 \times 10^{4}$ & $-2.15 \times 10^{4}$ & $-8.40 \times 10^{3}$ & $-1.78 \times 10^{4}$ \\
$M(\mathrm{~g})$ & 46.83 & 30.70 & 25.65 & 13.61 \\
$\gamma\left(\right.$ day $\left.^{-1}\right)$ & $1.65 \times 10^{-2}$ & $1.86 \times 10^{-2}$ & $1.97 \times 10^{-2}$ & $2.27 \times 10^{-2}$ \\
\hline
\end{tabular}

Equation 7 defines the integration limits in the case of a linear change in in utero RBC life span. Equation 3 then becomes (Fig. 2):

$n(0)=\int_{\frac{-L(0)}{1+\alpha}}^{0} R(t) \mathrm{d} t$

Furthermore, the number of RBCs produced in utero that are removed from circulation until time $t$ after birth is then given by (Fig. 2):

$n_{\mathrm{E}}(t)=\int_{\frac{-L(0)}{1+\alpha}}^{\frac{t-L(0)}{1+\alpha}} R(u) \mathrm{d} u$

The number of RBCs that were produced in utero that remain in circulation after birth at time $t$ can then be calculated as:

$n(t)=n(0)-n_{\mathrm{E}}(t)$

where $n(0)$ is the number of RBCs produced in utero that are present at the time of birth. If a small fraction of these RBCs are removed, labeled, and reinfused back into the same infant, then:

$n_{\mathrm{L}}(t)=F_{\mathrm{L}} \cdot n(t)$

where $n_{\mathrm{L}}(t)$ is the number of labeled RBCs produced in utero that remain in circulation after birth at time $t$, and $F_{\mathrm{L}}$ is the fraction of labeled RBCs relative to the total number of RBCs present. The amount of $\mathrm{Hb}$ present in the labeled RBCs produced in utero that remain in circulation after birth at time $t$ can be given as:

$\mathrm{Hb}_{\mathrm{L}}(t)=\mathrm{MCH} n_{\mathrm{L}}(t)$

where $\mathrm{MCH}$ is the mean corpuscular hemoglobin of the RBCs. Substituting Eqs. 1, 2, 8-11 in Eq. 12 and integrating the final model to calculate the amount of $\mathrm{Hb}$ present in labeled RBCs produced in utero and remaining in circulation after birth at time $t$ can be given as:

$\mathrm{Hb}_{\mathrm{L}}(t)= \begin{cases}F_{\mathrm{L}} \cdot \mathrm{MCH} \cdot k \cdot\left[\frac{M}{\gamma} \cdot S_{1}(t)+M \cdot S_{2}(t)+S_{3}\right] & t \leq(p-\mathrm{GA})(1+\alpha)+L(0) \\ F_{\mathrm{L}} \cdot \mathrm{MCH} \cdot k \cdot S_{4}(t) & L(0) \geq t>(p-\mathrm{GA})(1+\alpha)+L(0)\end{cases}$ where $p=154$ days (Eq. 1) and

$S_{1}(t)=e^{\gamma \cdot p}-e^{\gamma \cdot\left(\mathrm{GA}+\frac{t-L(0)}{1+\alpha}\right)}$

$S_{2}(t)=\mathrm{GA}-p+\frac{t-L(0)}{1+\alpha}$

$S_{3}=\left\{\begin{array}{cc}\frac{A}{5} \cdot\left(\mathrm{GA}^{5}-p^{5}\right)+\frac{B}{4} \cdot\left(\mathrm{GA}^{4}-p^{4}\right)+\frac{C}{3} \cdot\left(\mathrm{GA}^{3}-p^{3}\right)+\frac{D}{2} \cdot\left(\mathrm{GA}^{2}-p^{2}\right)+E \cdot(\mathrm{GA}-p) & \mathrm{GA} \geq p \\ 0 & \mathrm{GA}<p\end{array}\right.$

$$
\begin{aligned}
S_{4}(t)= & \frac{A}{5} \cdot\left(\mathrm{GA}^{5}-\left(\mathrm{GA}+\frac{t-L(0)}{1+\alpha}\right)^{5}\right) \\
& +\frac{B}{4} \cdot\left(\mathrm{GA}^{4}-\left(\mathrm{GA}+\frac{t-L(0)}{1+\alpha}\right)^{4}\right) \\
& +\frac{C}{3} \cdot\left(\mathrm{GA}^{3}-\left(\mathrm{GA}+\frac{t-L(0)}{1+\alpha}\right)^{3}\right) \\
& +\frac{D}{2} \cdot\left(\mathrm{GA}^{2}-\left(\mathrm{GA}+\frac{t-L(0)}{1+\alpha}\right)^{2}\right)+E \cdot\left(\frac{L(0)-t}{1+\alpha}\right)
\end{aligned}
$$

It is assumed that the disposition of $\mathrm{Hb} / \mathrm{RBCs}$ was life span based (i.e., RBCs were removed from circulation through cellular aging/senescence) (14-17). The model also assumed a single point distribution of RBC life spans, i.e., RBCs produced at a given time in utero have the same RBC life span.

\section{Accurately Accounting for Phlebotomies in the Analysis}

Newborn infants are subjected to multiple phlebotomies for clinical testing purposes; accordingly, Eq. 13 must be modified to accurately account for the perturbations in the $\mathrm{Hb}$ level caused by the phlebotomies. We accounted for the loss of labeled RBCs from circulation as previously described (18-20). Details of the phlebotomy correction are described in the Appendix. 


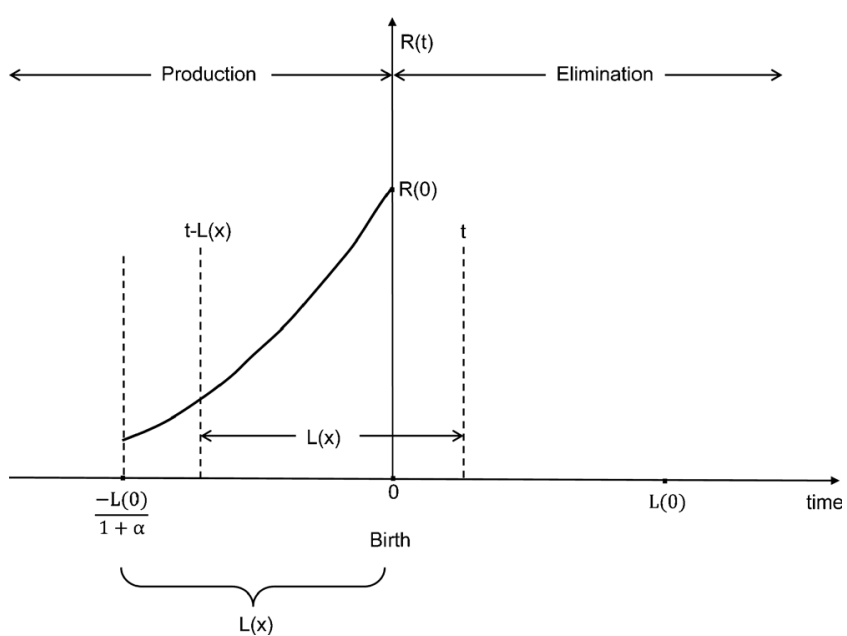

Fig. 2. Non-SS fetal erythropoiesis in newborn infants. The solid line represents the changes in fetal erythropoiesis rate up to the time of birth. $R(0)$ represents the RBC production rate at time of birth $(t=0)$. The fetal erythropoiesis rate is proportional to the in utero infant body weight (Eq. 2). Fetal RBC life span, $L(x)$, varies linearly with time (Eq. 3)

\section{Subjects}

Ten VLBW preterm infants between 24 and 28 weeks gestation being cared for in the Neonatal Intensive Care Unit (NICU) at the University of Iowa Children's Hospital were enrolled in this study. The study was approved by the University of Iowa Human Subject Internal Review Board. All subject's parents or legal guardians provided written informed consent as part of an ongoing consent process. Inclusion criteria included treatment with expectation of survival and moderate to severe respiratory distress requiring mechanical ventilation. Exclusion criteria included hematological diseases (except for anemia associated with phlebotomy loss and prematurity), alloimmune hemolytic anemia, diffuse intravascular coagulation, thrombosis, and transfusion requirements that were emergent and did not allow controlled sampling.

\section{Biotinylation of RBCs and FACs Analysis}

The measurement of red cell survival using RBCs labeled with biotin (BioRBCs) is practical, reliable, accurate, and safe (21-23). RBCs were labeled with biotin as previously described $(21,22)$. Briefly, RBCs from study subjects were washed twice and prepared at $25 \%$ hematocrit (Hct). The biotinylation reagent sulfo NHS-biotin was dissolved and used to label RBCs at a discrete biotin density. After a 30-min reaction, the BioRBCs were washed twice, filtered, and transfused. The percent of BioRBCs in posttransfusion blood samples was determined by flow cytometric enumeration after staining with avidin conjugated with Alexa Fluor 488 as previously described $(21,22)$. Cord blood/infant autologous RBCs were biotinylated as described earlier and reinfused back into the same infant. Each infant received only a single BioRBC transfusion during the study period. All BioRBC transfusions were administered within the first 2 days of life.

\section{BioRBC Survival}

Cord blood RBCs collected at the time of birth are comprised entirely of fetal RBCs. Autologous infant RBCs collected very close to the time of birth also are comprised almost entirely of fetal RBCs. RBC production falls several fold after birth, and thus, the number of RBC produced after birth within the first 2 days is only a very small fraction compared to the total number of fetal RBCs that are present in the infant at the time of birth. Because of this and due to the limited number of infants that receive cord blood RBC transfusions, this analysis also included infants that receive $\mathrm{RBC}$ transfusions from autologous RBCs taken within the first 2 days of birth.

Phlebotomy blood samples from birth through the end of the BioRBC study period were weighed and recorded immediately after collection. The blood collection tube weights were subtracted from the total weights and converted to the volume of blood removed based on a specific gravity of blood of 1.05 (24). The $\mathrm{Hb}$ mass removed with each phlebotomy was calculated by multiplying the volume of blood removed times the $\mathrm{Hb}$ concentration measured at the time of blood sampling. In addition to the BioRBC transfusion, the infants also received additional unlabeled $\mathrm{RBC}$ transfusions at various times based on the severity of anemia. The decision to treat with RBC transfusions was made by the physician in accordance with NICU guidelines (25). The volume of packed RBCs administered ( $85 \%$ Hct) and the time of RBC transfusions were recorded for use in the analysis. The MCH parameter was set equal to $37.5 \mathrm{pg} / \mathrm{cell}$ based on previous estimates $(18,19)$.

\section{Data Analysis}

The amount of $\mathrm{Hb}$ present in BioRBCs over time, $\mathrm{Hb}_{\mathrm{L}}(t)$ (Eq. 13), was modeled instead of the total number of BioRBCs over time, $n_{\mathrm{L}}(t)$ (Eq. 11). Flow cytometric analysis of BioRBCs is an enumeration technique. At each BioRBC sampling time, the fraction of BioRBCs relative to the total number of RBCs in the sample can be accurately estimated. To calculate the total absolute number of BioRBCs in the infant circulation at a particular sampling time, $n_{\mathrm{L}}(t)($ Eq. 11), this fraction has to be multiplied by the total number of infant RBCs in circulation at that sampling time. In this study, the total number of RBCs in circulation could not be measured at the time of each BioRBC sample. Instead, the $\mathrm{Hb}$ concentration measurements available for each BioRBC sampling time were used to estimate the absolute amount of $\mathrm{Hb}$ present in the BioRBCs in infant circulation over time (Eq. 13).

All modeling and simulations were conducted using WINFUNFIT, a Windows (Microsoft) version evolved from the general nonlinear regression program FUNFIT (26), using ordinary least squares fit to each individual subject's $\mathrm{Hb}$ amount-time profile. To characterize the uncertainty in the estimates of the individual subject parameters, the standard deviation (SD) and the percent coefficient of variation $(\mathrm{CV} \%)$ of the estimates were calculated for each parameter. 


\section{RESULTS}

\section{Subject Characteristics}

The mean GA of the ten newborn subjects was 180.7 days (range, 162 to 194). The mean birth weight was $0.815 \mathrm{~kg}$ (range, 0.564 to $1.250 \mathrm{~kg}$ ). Three males (all singletons) and seven females (six singletons and one twin) were studied. Of the ten infants, three received cord blood BioRBCs and seven received biotin-labeled autologous RBCs drawn within the first 2 days of birth. The infants underwent an average of 142 phlebotomies (range, 50 to 271). The average number of RBC transfusions was 4.7 (range, 1 to 12 ). For all transfusions administered, the volume of packed RBCs (85\% Hct) administered was $15 \mathrm{~mL} / \mathrm{kg}$.

\section{Model Simulations}

Figure 3 shows the effect of varying individual parameters $(\alpha, k$, and $L(0))$ and the effect of multiple clinical phlebotomies on the model-predicted BioRBC survival curves. Figure 3 a shows the effect of varying $\alpha$, the slope associated with the rate of change in fetal RBC life span (Eq. 4), on the simulated BioRBC
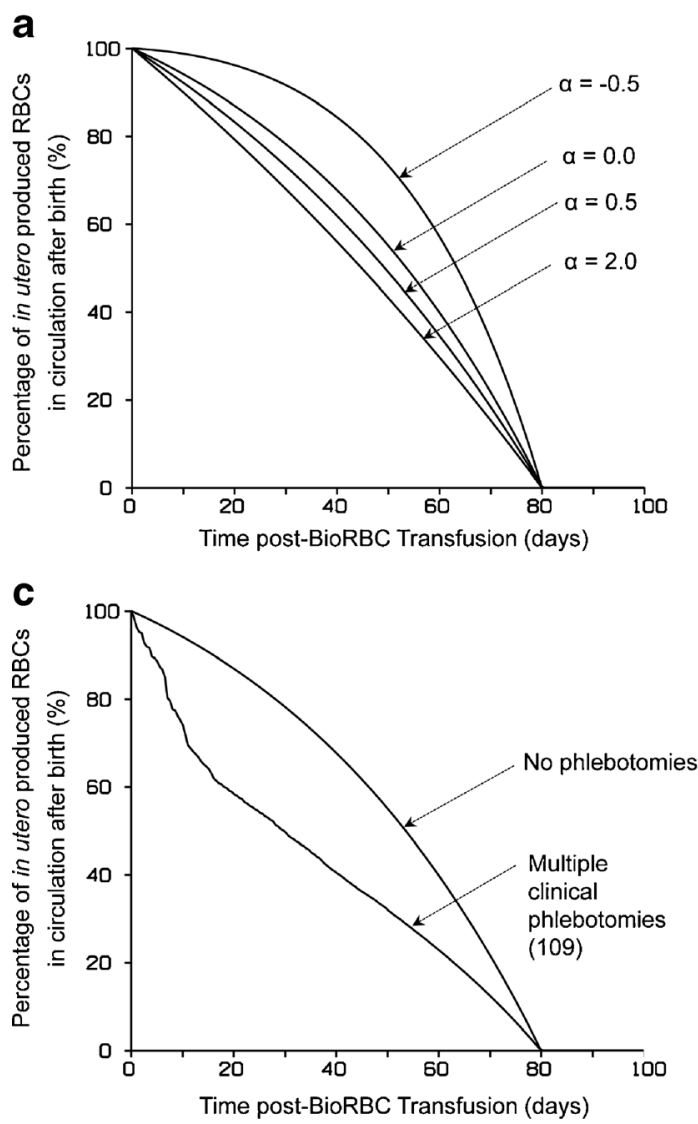

survival curve with all other model parameters fixed at specified values: $L(0)=80$ days; $k=0.60 \times 10^{8} \mathrm{RBC} /$ day g; no clinical phlebotomies. Similarly, Fig. $3 b$ shows the effect of varying $L(0)$, the RBC life span at the time of birth, on the simulated BioRBC survival curve with fixed parameters of $\alpha=0, k=0.60 \times 10^{8} \mathrm{RBCs} /$ day $\mathrm{g}$, and no clinical phlebotomies. Figure $3 c$ shows the effect of multiple clinical phlebotomies on the simulated BioRBC survival curve with fixed parameters of $L(0)=$ 80 days, $k=0.60 \times 10^{8} \mathrm{RBCs} /$ day g, and $\alpha=0$. Finally, the effect of varying $k$, the scaling factor associated with the fetal RBC production rate, on the model-predicted BioRBC survival curve is depicted in Fig. 3d with fixed parameters of $\alpha=0, L(0)=80$ days, and no clinical phlebotomies.

\section{Model Fit to Infant Data}

The model (Eq. 13) fit to the Hb amount-time profiles, along with the cumulative amount of $\mathrm{Hb}$ removed for four representative subjects, is displayed in Fig. 4. General agreement between the model fit and the $\mathrm{Hb}$ amount data was observed. The estimates of the parameters are summarized in Table II.

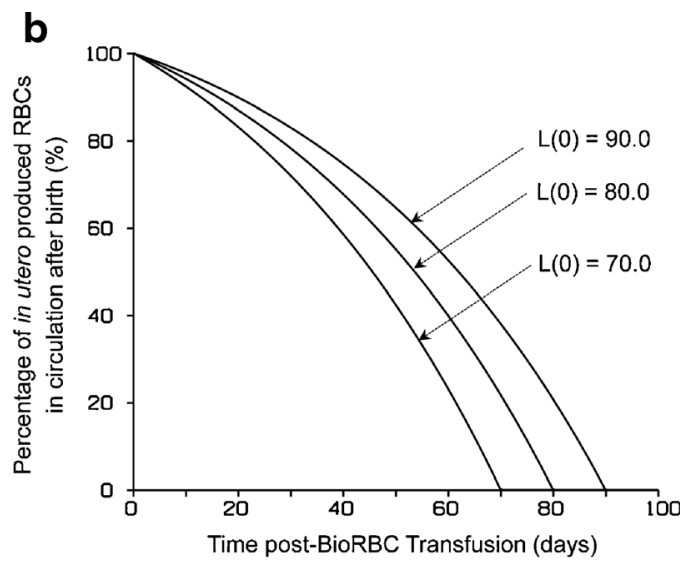

d

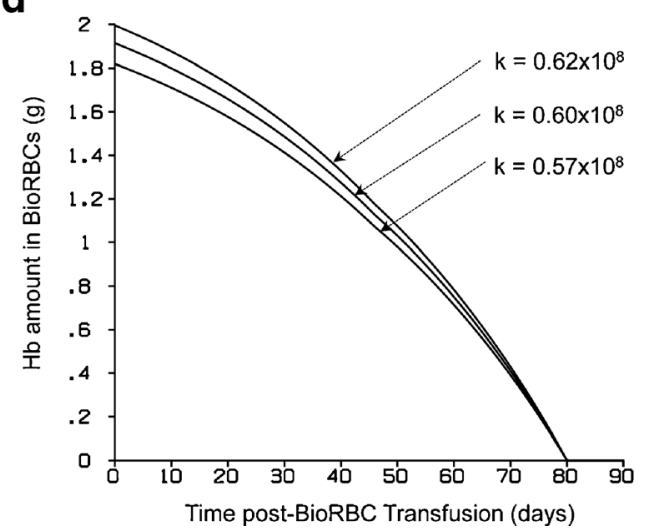

Fig. 3. Model simulated cord blood RBC survival curves. a The effect of varying $\alpha$ (Eq. 5), on the simulated RBC survival curve. All other model parameters were fixed at specified values $\left(L(0)=80\right.$ days, $k=0.60 \times 10^{8} \mathrm{RBCs} / \mathrm{day} \mathrm{g}$ with no clinical phlebotomies). b The effect of varying $L(0)$ on the simulated RBC survival curve $(\alpha=0.0, k=0.60 \times$ $10^{8} \mathrm{RBCs} /$ day g with no clinical phlebotomies). $\mathbf{c}$ The effect of multiple clinical phlebotomies on the simulated RBC survival curve $\left(L(0)=80\right.$ days, $k=0.60 \times 10^{8} \mathrm{RBCs} /$ day $\mathrm{g}$ and $\left.\alpha=0.0\right)$. The effect of varying $k$ on the model-predicted $\mathrm{RBC}$ survival curve can be observed in $\mathbf{d}(\alpha=0.0, L(0)=80$ days with no clinical phlebotomies) 
a

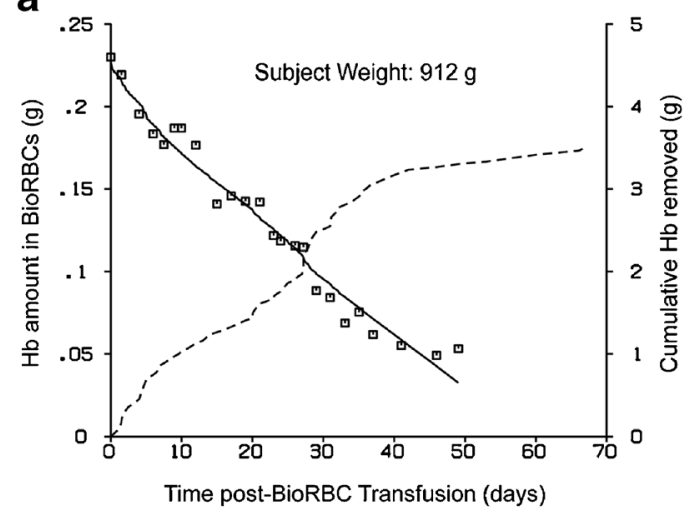

C

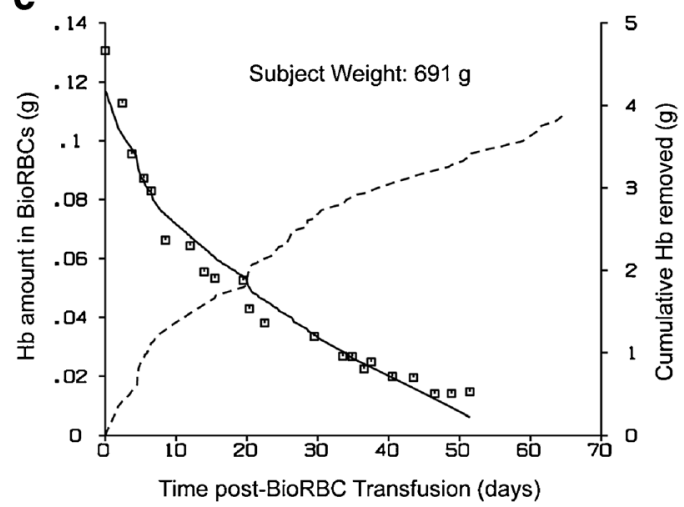

b

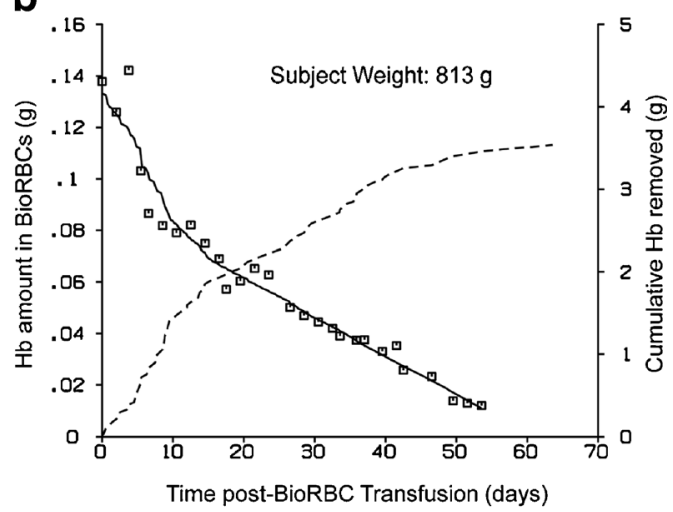

d

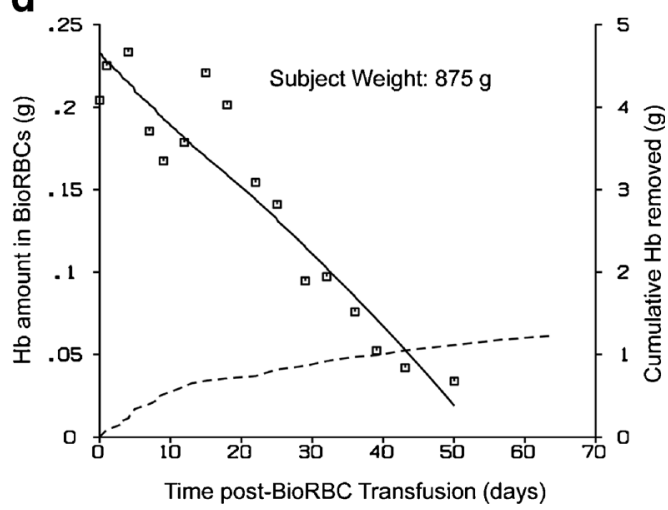

Fig. 4. a-d Model fit to $\mathrm{Hb}$ amount-time data for four subjects. The open squares represent $\mathrm{Hb}$ amount data points and the solid line shows the model fit (Eq. 10). The dashed line represents the cumulative amount of Hb removed from the infant during the same time interval

\section{DISCUSSION}

This study introduces a novel method for utilizing the in vivo disappearance of cord blood RBCs of newborn infants labeled ex vivo to evaluate fetal erythropoiesis. Although RBC survival curves have been used previously for estimating fetal RBC life span, the use of these data for evaluating fetal erythropoiesis has not been previously described.

\section{Model Simulations}

The effect of individual parameters $(\alpha, k$, and $L(0))$ and the effect of multiple clinical phlebotomies on the modelpredicted BioRBC survival curves are displayed in Fig. 3. Parameter $\alpha$ describes the rate of change (slope) in fetal RBC life span with time (Eq. 4). When $\alpha$ is 0 (Fig. 3a), the fetal $\mathrm{RBC}$ life span is fixed in utero, i.e., the fetal RBC life span does not change with development during this time. When $\alpha$ is negative, the model assumes that the fetal RBC life span decreases with fetal development and approaches $L(0)$, the life span at time of birth $(t=0)$. Finally, when $\alpha$ is positive, the model assumes that the fetal RBC life span increases with fetal development and approaches the RBC life span at the time of birth. The last case is likely the most physiologically relevant because previous studies are consistent with the inference that infant RBCs have shorter life spans than those of healthy adults and that RBC life span is shorter in preterm infants than in term infants $(2,9,10)$.
$L(0)$ describes the RBC life span at the time of infant birth $(t=0)$. Since the model describes the survival of cord blood RBCs, the RBCs produced at the time of birth are the youngest RBCs in the collected cord blood sample. When this sample is labeled and reinfused back into the same infant, the youngest RBCs will remain in the circulation the longest. Thus, $L(0)$ represents the time the youngest RBCs in the population will survive. For example, if $L(0)$ was 80 days, then the youngest labeled cord blood RBCs will be removed from circulation 80 days after these cells where introduced into the circulation. Beyond 80 days, none of the labeled RBCs will be present in infant circulation. Thus, $L(0)$ determines the end point of the RBC survival curve (Fig. 3b).

Newborn infants, especially critically ill preterm anemic low birth infants, are subjected to multiple clinical blood sampling as part of their routine care and management. Any phlebotomy following the transfusion of labeled RBCs will perturb the RBC survival curve. This perturbation becomes increasingly significant as infants are subjected to more and

Table II. Estimated Parameter Summary from the $\mathrm{Hb}$ Mass Model $(n=10)$

\begin{tabular}{lccc}
\hline & $\alpha($ days/day $)$ & $L(0)($ day $)$ & $k\left(\times 10^{7}\right.$ RBCs/day g) \\
\hline Mean & 0.5076 & 52.06 & 1.07 \\
SD & 0.0648 & 10.80 & 0.51 \\
CV $(\%)$ & 12.76 & 20.75 & 47.75 \\
\hline
\end{tabular}

$S D$ standard deviation, $C V$ coefficient of variation 
more phlebotomies. Each phlebotomy removes a certain fraction of the labeled RBCs from the circulation resulting in a decline in RBC survival curve. As shown in Fig. 3c, the effect of 109 clinical phlebotomies on labeled RBCs significantly affected the shape of the model-predicted RBC survival curve.

Finally, $k$ is the scaling parameter that relates the in utero growth of the infant to the rate of fetal erythropoiesis (Eq. 2). When all the other factors in the model are kept the same (i.e., same GA, gender, singleton/twin), a larger value of $k$ indicates that the infant has a higher fetal erythropoiesis rate as compared to an infant with lower $k$ value (Fig. 3d).

\section{Model Fit to Infant data}

The applicability of the model has been described using an example dataset of cord blood/autologous RBCs from ten VLBW preterm infants that were labeled with biotin. The model successfully described the elimination of cord blood/ autologous BioRBCs in these infants (Fig. 4) and accounted for (1) all blood removed and transfused and (2) an increase in body weight due to infant growth and blood volume expansion.

A positive value for $\alpha$ indicates that the fetal RBC life span increases with fetal development. In the present study, the mean model estimate for $\alpha$ of 0.5076 (Table II) suggests that fetal RBC life span increased at a rate of $\sim 0.51$ day/day gestation in the ten subjects, and is similar to what has been previously reported in ovine fetuses (10).

The mean RBC life span at the time of birth, $L(0)$, was 52.06 days (Table II). This is similar to the previous range of $\mathrm{RBC}$ life span estimates of 35 to 50 days based on ${ }^{51} \mathrm{Cr}$ labeled RBCs (27). As expected, the estimated infant RBC life spans were shorter than the reported adult RBC life span of 120 days (28). The reduced fetal $\mathrm{RBC}$ life span as compared to adult RBC life span may be due to the differences in the conditions under which these RBCs were produced. RBCs in healthy adults are produced under steadystate conditions. In contrast, fetal RBCs are produced under hypoxic intrauterine conditions during a period of rapid increase in the number of circulating RBCs. This results in a forced accelerated maturation of fetal RBCs and thus resembles "stress erythropoiesis" of later life.

The mean body weight-scaled fetal RBC production rate, $k$, of $1.07 \times 10^{7} \mathrm{RBCs} /$ day $\mathrm{g}$ (which corresponds to an erythropoiesis rate of $1.07 \times 10^{10} \mathrm{RBCs} /$ day in a 1000 -g infant) was similar to previously reported in utero $\mathrm{Hb}$ stimulation rate of $0.414 \mathrm{~g} / \mathrm{day}_{\mathrm{kg}}^{3 / 4}$ (which corresponds to $1.104 \times 10^{10}$ $\mathrm{RBCs} /$ day in a 1000-g infant) (18). The estimated in utero erythropoiesis rate was also approximately threefold higher than the RBC production rate after birth (18). Previous studies of bone marrow, reticulocytes, and iron kinetics have unequivocally substantiated this drop in erythropoiesis after birth (8).

\section{Clinical Significance}

Due to practical and ethical concerns with fetal blood sampling, there is very limited information available on the dynamic changes associated with in utero RBC production. This study introduces a novel method to utilize cord blood RBCs collected at the time of birth to look "backward in time" to evaluate fetal erythropoiesis. The proposed model can be used to study fetal erythropoiesis under nonsteadystate conditions while also accounting for in utero changes in fetal RBC life span. Given the gestational age of a newborn infant, this model can be used to estimate the RBC production rate at the time of birth and also to estimate how soon the RBCs present at the time of birth are removed from infant circulation after birth. The data derived from this study are clinically relevant in that they enhance understanding fetal and neonatal anemia and can help to guide the evaluation of therapeutic interventions in the future.

\section{Limitations of the Model}

The proposed model assumes that the fetal RBC life span varies linearly with GA. Although this assumption is based on previously published studies in ovine fetuses (10), it has yet to be verified in humans. Due to obvious ethical and regulatory concerns with fetal sampling, there is limited information regarding the intrauterine changes in human fetal RBC life span. The aim of the proposed model was to introduce a novel approach of utilizing cord blood RBC survival data to better understand fetal erythropoiesis. A similar model to that which we proposed here can be derived for nonlinear intrauterine changes in $\mathrm{RBC}$ life span with gestation.

The model also assumes that the fetal erythropoiesis rate is a function of the in utero growth of the fetus. During the latter part of fetal life, the fetus grows rapidly and there is a rapid increase in fetal weight. This increases the demand for oxygenation of the fetal tissues and must be met by a proportional increase in in utero $\mathrm{RBC}$ production rate. Further experimental evidence is needed to validate this assumption.

\section{CONCLUSION}

The present study for the first time demonstrates a novel and versatile method for utilizing labeled cord blood RBCs of newborn infants to study non-SS fetal erythropoiesis. This method also accounts for changes in fetal RBC life span with GA. The model was successfully applied to cord blood/autologous BioRBC survival data from ten VLBW preterm anemic infants. The estimated parameters of the model were consistent with previously reported literature values, further supporting the utility of this model. Future investigations that study a greater number of infants encompassing a greater GA spectrum are needed to allow for identification of associations between the rate of fetal erythropoiesis and important factors influencing fetal erythropoiesis and fetal RBC life span.

\section{ACKNOWLEDGMENTS}

The authors express appreciation to Mark A. Hart for editorial and secretarial assistance. Also appreciated are the many outstanding clinical research contributions of Iowa's neonatal research nurse team who included Gretchen Cress, R.N., M.P.H.; Karen Johnson, R.N.; Gin Zhou, R.N.; and Ruthann Schrock, R.N. The gracious help provided by the 
University of Iowa clinical laboratory staff led by Mitchell J. Owen, MT (ASCP), and Mary Capper, MT (ASCP)SH, and overseen by Matthew D. Krasowski, M.D., Ph.D., was essential in the success of this study. This work would not have been possible without the permission of the parents of study subjects to allow their infants to participate. Finally, we are grateful to the Sysmex Corporation, Kobe, Japan (http:// www.sysmex.co.jp/en/index.html) for the generous loan of the Sysmex XE-2100 automatic hematology analyzer used in this study. This work was supported by the National Institutes of Health (NIH) US Public Health Service Program Project Grant 2 P01 HL046925 and the National Center for Research Resources, a part of the NIH, Grant Number UL1TR000039.

Conflict of Interest No conflicts of interest, financial or otherwise, are declared by the authors.

Author Contributions D.N., R.L.S., and J.A.W. performed the experiments; D.J.K., R.L.S., D.N., and J.A.W. analyzed the data. D.J.K. prepared the initial draft of the manuscript. P.V-P, D.N., R.L.S., J.A.W., and D.M. provided additional ideas and discussions and helped in the critical review. All authors approved the final version of the manuscript.

\section{GLOSSARY}

$\begin{array}{ll}\text { RBC } & \text { Red blood cell } \\ \mathrm{Hb} & \text { Hemoglobin } \\ \text { VLBW } & \text { Very low birth weight infant } \\ \mathrm{GA} & \text { Gestational age } \\ \text { Non-SS } & \text { Nonsteady state } \\ \text { BioRBC } & \text { Biotinylated RBC } \\ \mathrm{MCH} & \text { Mean corpuscular hemoglobin } \\ \mathrm{Hct} & \text { Hematocrit } \\ F_{\mathrm{L}} & \text { Fraction of labeled RBCs } \\ R(t) & \text { In utero erythropoiesis rate } \\ n(0) & \text { Number of RBCs produced in utero that are } \\ n_{\mathrm{E}}(t) & \text { present in circulation at birth } \\ n(t) & \text { Number of RBCs produced in utero that are } \\ \alpha & \text { eliminated from circulation at time } t \\ \mathrm{PCF} & \text { in circulation at time } t \\ L(0) & \text { Rate of change in in utero fetal RBC life span } \\ F_{\mathrm{RM} i} & \text { Life span (days) of RBCs present at birth }(t=0) \\ \mathrm{Hb} \mathrm{T}_{\mathrm{T}}(t) & \text { Fraction of total Hb remaining after } i^{\text {th }} \\ t & \text { phlebotomy } \\ & \text { Total hemoglobin (grams) at time } t\end{array}$

\section{APPENDIX}

\section{Correction for Phlebotomies}

Let us consider the $i$ th phlebotomy was performed at time $t_{\mathrm{P} i}$ that removed a certain fraction of $\mathrm{Hb}$ from circulation. The fraction remaining after the $i$ th phlebotomy, $F_{\mathrm{RM} i}$, is given by Eq. 18 :
$F_{\mathrm{RM} i}=\frac{\mathrm{Hb}_{\mathrm{T}}\left(t_{\mathrm{P} i}\right)-\mathrm{Hb}_{\mathrm{RM} i}}{\mathrm{Hb}_{\mathrm{T}}\left(t_{\mathrm{P} i}\right)}$,

where $\mathrm{Hb}_{\mathrm{RM} i}$ is the hemoglobin removed due to the $i$ th phlebotomy at time $t_{\mathrm{P} i}$. For multiple phlebotomies, the phlebotomy correction factor (PCF) can be calculated as shown in Eq. 19,

$\mathrm{PCF}=\left\{\begin{array}{l}q \\ \prod_{i=j} F_{\mathrm{RM} i} \text { if } q \geq j \text { and } t_{\mathrm{P} i}<t 1 \text { otherwise }\end{array}\right.$

where the fraction remaining after each phlebotomy is ordered from the first to the last phlebotomy, $j$ is the first phlebotomy after entry of the RBCs into the systemic circulation, and $q$ is the last phlebotomy prior to the current time $t$. In this case, $j$ represents the first phlebotomy after the BioRBCs are introduced into the circulation, and thus, Eq. 19 now can be written as Eq. 20. The derived PCF is then multiplied with Eq. 13 to account for loss of labeled RBCs due to multiple clinical phlebotomies.

$\mathrm{PCF}=\left\{\begin{array}{l}q \\ \prod_{i=1} F_{\mathrm{RM} i} \text { if } q \geq 1 \text { and } t_{\mathrm{P} i}<t 1 \text { otherwise }\end{array}\right.$

\section{REFERENCES}

1. Kett JC. Anemia in infancy. Pediatr Rev. 2012;33(4):186-7.

2. Kaplan E, Hsu KS. Determination of erythrocyte survival in newborn infants by means of Cr51-labelled erythrocytes. Pediatrics. 1961;27(3):354-61.

3. Sturgeon P. Volumetric and microscopic pattern of bone marrow in normal infants and children: II. Cytologic pattern. Pediatrics. 1951;7(5):642-50.

4. Bratteby L-E, Garby L, Wadman B. Studies on erythro-kinetics in infancy: XIII. The mean life span and the life span frequency function of red blood cells formed during foetal life. Acta Paediatr. 1968;57(4):305-10.

5. Palis J, Segel G. Developmental biology of erythropoiesis. Blood Rev. 1998;12(2):106-14.

6. Thomas DB, Yoffey J. Human foetal haemopoiesis I. The cellular composition of foetal blood. $\mathrm{Br} \mathrm{J}$ Haematol. 1962;8(3):290-5.

7. Walker J, Turnbull EN. Hæmoglobin and red cells in the human foetus: and their relation to the oxygen content of the blood in the vessels of the umbilical cord. Lancet. 1953;262(6781):312-8.

8. Finne P, Halvorsen S. Regulation of erythropoiesis in the fetus and newborn. Arch Dis Child. 1972;47(255):683.

9. Pearson HA. Life-span of the fetal red blood cell. J Pediatr. 1967;70(2):166-71.

10. Brace RA, Langendörfer C, Song T-B, Mock DM. Red blood cell life span in the ovine fetus. Am J Physiol Regul Integr Comp Physiol. 2000;279(4):R1196-204.

11. Hollingsworth JW. Lifespan of fetal erythrocytes. J Lab Clin Med. 1955;45(3):469-73.

12. Bratteby L-E, Garby L, Groth T, Schneider W, Wadman B. Studies on erythro-kinetics in infancy: XIII. The mean life span and the life span frequency function of red blood cells formed during foetal life. Acta Paediatr. 1968;57(4):311-20.

13. Arbuckle TE, Wilkins R, Sherman GJ. Birth weight percentiles by gestational age in Canada. Obstet Gynecol. 1993;81(1):39-48.

14. Krzyzanski W, Ramakrishnan R, Jusko W. Basic pharmacodynamic models for agents that alter production of natural cells. J Pharmacokinet Biopharm. 1999;27(5):467-89. 
15. Krzyzanski W, Woo S, Jusko W. Pharmacodynamic models for agents that alter production of natural cells with various distributions of lifespans. J Pharmacokinet Pharmacodyn. 2006;33(2):125-66.

16. Landaw SA. Factors that accelerate or retard red blood cell senescence. Blood Cells. 1988;14(1):47-67.

17. Freise K, Widness J, Schmidt R, Veng-Pedersen P. Modeling time variant distributions of cellular lifespans: increases in circulating reticulocyte lifespans following double phlebotomies in sheep. J Pharmacokinet Pharmacodyn. 2008;35(3):285-323.

18. Freise KJ, Widness JA, Veng-Pedersen P. Erythropoietic response to endogenous erythropoietin in premature very low birth weight infants. J Pharmacol Exp Ther. 2010;332(1):229-37.

19. Saleh MI, Nalbant D, Widness JA, Veng-Pedersen P. Population pharmacodynamic analysis of erythropoiesis in preterm infants for determining the anemia treatment potential of erythropoietin. 2013 2013-05-01 00:00:00. R772-R81 p.

20. Saleh MI, Widness JA, Veng-Pedersen P. Pharmacodynamic analysis of stress erythropoiesis: change in erythropoietin receptor pool size following double phlebotomies in sheep. Biopharm Drug Dispos. 2011;32(3):131. doi:10.1002/bdd.743.

21. Mock DM, Widness JA, Strauss RG, Franco RS. Posttransfusion red blood cell (RBC) survival determined using biotin-labeled
RBCs has distinct advantages over labeling with 51Cr. Transfusion (Paris). 2012;52(7):1596.

22. Mock DM, Matthews NI, Zhu S, Strauss RG, Schmidt RL, Nalbant D, et al. Red blood cell (RBC) survival determined in humans using RBCs labeled at multiple biotin densities. Transfusion (Paris). 2011;51(5):1047-57.

23. Kuruvilla DJ, Nalbant D, Widness JA, Veng-Pedersen P. Mean remaining life span: a new clinically relevant parameter to assess the quality of transfused red blood cells. Transfusion (Paris). 2014;54(10pt2):2724-9.

24. Trudnowski RJ, Rico RC. Specific gravity of blood and plasma at 4 and $37^{\circ} \mathrm{C}$. Clin Chem. 1974;20(5):615-6.

25. Strauss RG. How I transfuse red blood cells and platelets to infants with the anemia and thrombocytopenia of prematurity. Transfusion (Paris). 2008;48(2):209-17.

26. Veng-Pedersen P. Curve fitting and modeling in pharmacokinetics and some practical experiences with NONLIN and a new program FUNFIT. J Pharmacokinet Biopharm. 1977;5(5):513-31.

27. Brugnara C, Platt OS. The neonatal erythrocyte and its disorders. In: Nathan and Oski's hematology of infancy and childhood. 2003;1:36-66.

28. Kruse A, Uehlinger DE, Gotch F, Kotanko P, Levin NW. Red blood cell lifespan, erythropoiesis and hemoglobin control. Contrib Nephrol. 2008;161:247-54. 